\title{
Novel High-Quality Compact Microstrip Resonator and its Application to Bandpass Filter
}

\author{
B. A. Belyaev, A. M. Serzhantov, A. A. Leksikov, Ya F. Bal'va, and An A. Leksikov
}

\begin{abstract}
A novel compact microstrip resonator based on interdigital structure is proposed. The resonator has several times higher quality factor than similar resonators presented earlier. It is shown that the size of the resonator can be significantly reduced with substantial increase in quality factor by incrementing the number of pins in the interdigital structure. A 4-pole bandpass filter with central frequency $900 \mathrm{MHz}$ and an overall size $4.6 \mathrm{~mm}$ $\times 10.0 \mathrm{~mm}$ was designed and fabricated on the base of the proposed structure. The measured upper stopband of the filter was found to be up to $7.4 f_{\mathrm{o}}$ at the level $-40 \mathrm{~dB}$.
\end{abstract}

Index Terms-Bandpass filter (BPF), interdigital resonator, microstrip, microstrip resonator.

\section{INTRODUCTION}

A new generation of microwave radio systems impose specific constraints on the overall size of its components, particularly on bandpass filters (BPFs). For this reason, in the last decade, a huge amount of work has been dedicated to research on compact resonators and methods of reducing the size of the filters [2]-[11]. Starting from simple stepped-impedance [1] and spiral resonators [2], progress brings them to complex hairpin and interdigital structures [3]-[6]. However, it is important to note that all these methods for miniaturization lead to a substantial decrease in unloaded quality factors of the resonators, and, hence, to the deterioration in the frequency-selective properties of the BPFs formed by the resonators. It is well known that the best unloaded quality factor among microstrip resonators has the one without any irregularity in its impedance. Another way to decrease the size of a resonator is to make stripline multilayer structure with number of dielectric layers more than 2 [12]. These structures have sufficiently higher quality factor, but at the same time they are much more complicated in fabrication and tuning. So, it is desirable to find a compact microstrip structure without irregularities in strip conductors forming it.

In this letter, a new design of a compact microstrip resonator is proposed, which has the highest magnitude of its unloaded quality factor among the known analogues having the same frequency and size.

Manuscript received September 10, 2014; revised December 18, 2014, February 11, 2015, and April 30, 2015; accepted June 10, 2015. This work was supported by the Ministry of Education and Science of the Russian Federation under State contract 14.607.21.0039.

B. A. Belyaev, A. A. Leksikov, Y. F. Bal'va, and A. A. Leksikov are with the Kirensky Institute of Physics SB RAS, Krasnoyarsk, 660036, Russia (e-mail: a.a.leksikov@gmail.com).

A. M. Serzhantov is with the Siberian Federal University, Krasnoyarsk, 660074, Russia (e-mail: cubicus@mail.ru).

Digital Object Identifier 10.1109/LMWC.2015.2451363

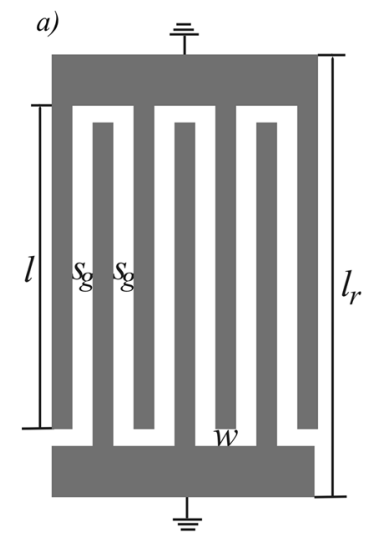

b)

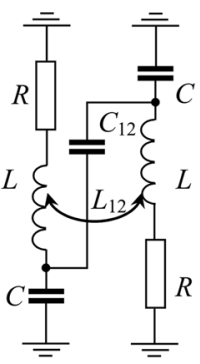

Fig. 1. Topology of the resonator (a) and equivalent circuit in the case of only two pins in the structure (b).

In Section II, the theory of the resonator and its comparison with similar resonators are presented. A 4-pole BPF with $20 \%$ bandwidth based on the resonator that was designed and fabricated is described in Section III.

\section{RESONATOR'S THEORY}

The topology of the resonator is presented in Fig. 1(a). It consists of two comb structures directed opposite to each other, thereby forming the so-called interdigital structure. The main difference between the resonator proposed and the resonators presented earlier [3], [4], [10] is that the common conductor of each comb structure is connected to the ground over its entire length, whereas in the prototype resonators, they are connected to each other by a narrow microstrip conductor. Each pin in the structure can be considered as a quarter-wave resonator. In the case when the width of the pins and gaps between them are very small when compared with the substrate thickness, the electromagnetic coupling between them is very strong. Moreover, high-frequency currents of the first mode with lowest frequency $f_{1}$ in the pins flow in the same direction while the voltage on the adjacent pins has different signs. Thus, a summation of the inductive and capacitive coupling takes place and one can observe significant repulsion of the eigenfrequencies of the resonator that leads to significant lowering of the first-mode frequency.

The equivalent circuit of the resonator is presented in Fig. 1(b). To simplify the analysis, a resonator consisting of only two pins will be considered. Each of the pins may be represented as an oscillation circuit formed by an inductance $L$, a capacitance $C$, and a resistance $R$ to account for the loss in the circuit. The coupling between the pins is characterized by mutual capacitance $C_{12}$ and mutual inductance $L_{12}$. A 
resonance frequency of the oscillation circuit can be calculated as $f_{0}=1 / 2 \pi \sqrt{L C}$ and its unloaded quality factor as $Q_{0}=(1 / R) \sqrt{L / C}$.

The lower $f_{\mathrm{e}}$ and upper $f_{\mathrm{o}}$ resonant frequencies of the shown resonant structure were calculated using Kirchhoff's equation

$$
f_{e}=\frac{1}{2 \pi \sqrt{\left(L+L_{12}\right)\left(C+2 C_{12}\right)}}, f_{o}=\frac{1}{2 \pi \sqrt{\left(L-L_{12}\right) C}} .
$$

It should be noted that at lower frequency $f_{\mathrm{e}}$, the currents in the neighboring pins flow in the same direction, but their potentials (on the capacitor plates of $C_{12}$ ) have opposite signs. At the same time, at the upper frequency $f_{\mathrm{o}}$, the situation is vice versa.

It is easy to show that at the first resonant frequency of a pair of coupled loops, they can be replaced by a single loop with inductance $L_{1}$, capacitor $C_{1}$, and resistance $R_{1}$, where

$$
L_{1}=\frac{1}{2}\left(L+L_{12}\right), C_{1}=2\left(C+2 C_{12}\right), R_{1}=\frac{R}{2} .
$$

In the case of microstrip line based on the substrate with high dielectric permittivity, when the width of the strips and the gap between them are much smaller than the substrate thickness, one can use the following simplification:

$$
C_{12} \ll C, L_{12} \approx L
$$

to calculate in quasi-static approximation the line parameters. As a result, equivalent inductance of the circuit consisting of two opposing pins is close by its value to the inductance of a single pin, while the equivalent capacitance is two times higher and the resistance reduces twice.

Similar calculations performed for the cases with number of pins 3, 4, and higher give an analytical expression for the unloaded quality factor of the lowest resonance and its frequency

$$
Q_{N}=\sqrt{\frac{N}{2}} Q_{2}, f_{N}=\frac{1}{\sqrt{\frac{N}{2}}} f_{2}(N \geq 2)
$$

where $Q_{2}$ and $f_{2}$ are the unloaded quality factor and resonant frequency of the interdigital structure with two pins and $N$ is the number of pins in the structure. Earlier [13] the expressions were derived for the case when $N \geq 1$, but when $N=1$ the structure cannot be considered as an interdigital one.

One can see from the expressions (3) that growth in the number of pins in the structure brings to the growth in the resonator unloaded Q-factor with simultaneous reduction of its resonant frequency. It will be recalled that the expressions presented above are suitable only in the case when the width of the pins and the gap between them are much smaller than the substrate thickness.

The conclusion drawn on the basis of interdigital structure's equivalent circuit was approved by electromagnetic simulation in AWR Design Environment and Sonnet Studio Suite. A dielectric substrate with $\varepsilon=80, \tan \delta=0.0003$ and a thickness $h=1 \mathrm{~mm}$ was chosen as base for the copper resonator $\left(\rho=5.88 \times 10^{7} \mathrm{Sm} / \mathrm{m}\right)$ having length and width of the pins as $4.4 \mathrm{~mm}$ and $100 \mu \mathrm{m}$ relatively, the gap between them being $20 \mu \mathrm{m}$. These correspond to $L=8.12 \times 10^{-10} \mathrm{H} / \mathrm{mm}, L_{12}=$ $5.6 \times 10^{-10} \mathrm{H} / \mathrm{mm}, C=3.02 \times 10^{-13} \mathrm{~F} / \mathrm{mm}, C_{12}=7.06 \times$ $10^{-13} \mathrm{~F} / \mathrm{mm}$. The box was chosen without top cover. The result of the simulation, particularly the dependence of the unloaded

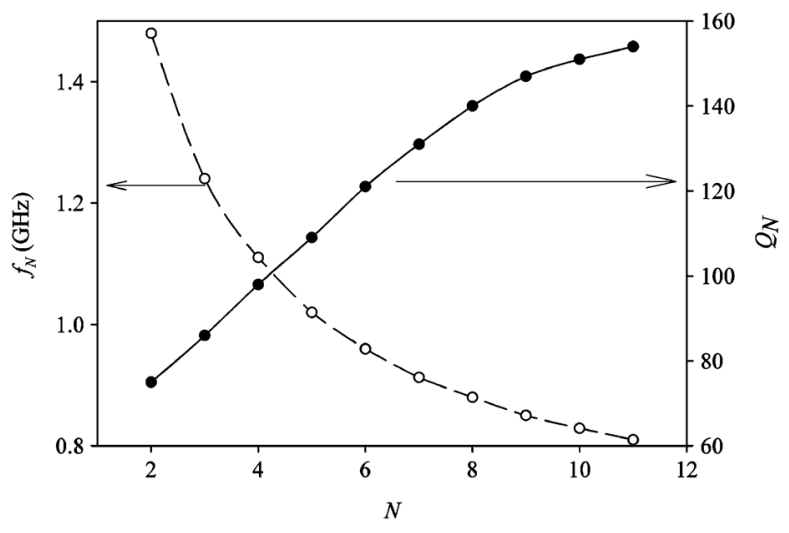

Fig. 2. Dependence of the resonant frequency and unloaded quality factor versus number of pins $N$ in the structure.

TABLE I

COMPARISON OF MICROSTRIP RESONATORS

\begin{tabular}{llc}
\hline \hline Ref. & \multicolumn{1}{c}{ Resonator type } & $\begin{array}{c}\text { Unloaded quality } \\
\text { factor }\end{array}$ \\
\hline$[2]$ & Spiral resonator & 33 \\
{$[3]$} & Interdigital structure & 24 \\
{$[4]$} & Interdigital structure & 48 \\
{$[7]$} & Hairpin quarter-wave & 31 \\
{$[10]$} & Capacitively loaded loop resonator & $<20$ \\
This work & Interdigital structure & 120 \\
\hline \hline
\end{tabular}

quality factor versus the number of pins and dependence of resonant frequency versus $N$ are presented in Fig. 2. It can be seen that frequency $f_{1}$ is significantly reduced and quality factor $Q_{1}$ increases with the number of pins $N$ in the interdigital structure.

To compare the presented resonator with the most compact microstrip resonators described earlier [2]-[4], [7], [10], their electromagnetic simulation was done for the resonant frequency $f_{1}=820 \mathrm{MHz}$ with the fixed area of the conductor topology $\left(1 \times 5.5 \mathrm{~mm}^{2}\right)$. The chosen substrate in all the resonators had $\varepsilon=80$ and a thickness $h=1 \mathrm{~mm}$. The results of the simulation are presented in Table I. One can see that the proposed resonator has $Q$-factor at least 2.5 times higher than the resonators proposed earlier.

The result can be explained as follows: the high-frequency currents in the pins of interdigital structure flow in the same direction; this is why the summary inductance of all the conductors has nearly the same magnitude as the inductance of a single conductor. At the same time, the current in the resonator is shared uniformly onto all the pins, thereby reducing resistive loss in the resonator.

\section{4-POLE BANDPASS FILTER}

A 4-pole BPF having 20\% fractional bandwidth was designed and fabricated by means of photolithography, it's topology is presented in Fig. 3. The same substrate with $\varepsilon=80$ and a thickness $h=1 \mathrm{~mm}$ was chosen as base for the filter. The lateral size of the filter was found to be $10 \mathrm{~mm} \times 4.6 \mathrm{~mm}$ that corresponds to $0.0303 \lambda_{g} \times 0.0132 \lambda_{g}$ for the central frequency $900 \mathrm{MHz}$. The topology parameters were as follows: length of the pins $l=3.9 \mathrm{~mm}$, width of the pins $w=120 \mu \mathrm{m}$, the gap between them $s_{g}=20 \mu \mathrm{m}$, full length of the copper resonators $l_{r}=4.6 \mathrm{~mm}$. The pins in the inner resonators were curtailed on $100 \mu \mathrm{m}$. The gap between inner resonators was $1.46 \mathrm{~mm}$ and that between inner and outer resonators $1.12 \mathrm{~mm}$. A galvanic 


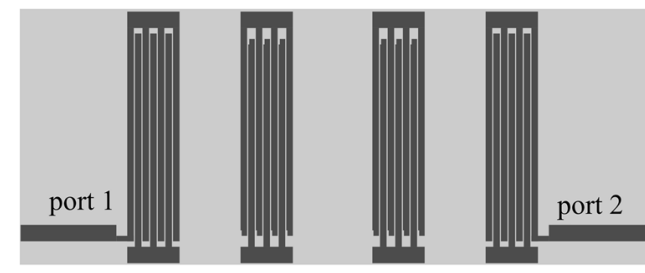

Fig. 3. A topology of the designed BPF.

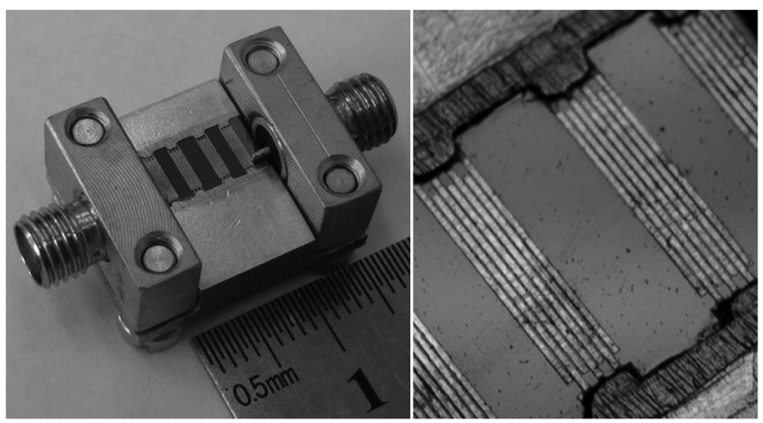

Fig. 4. Photograph of the fabricated filter and magnification of the resonators.
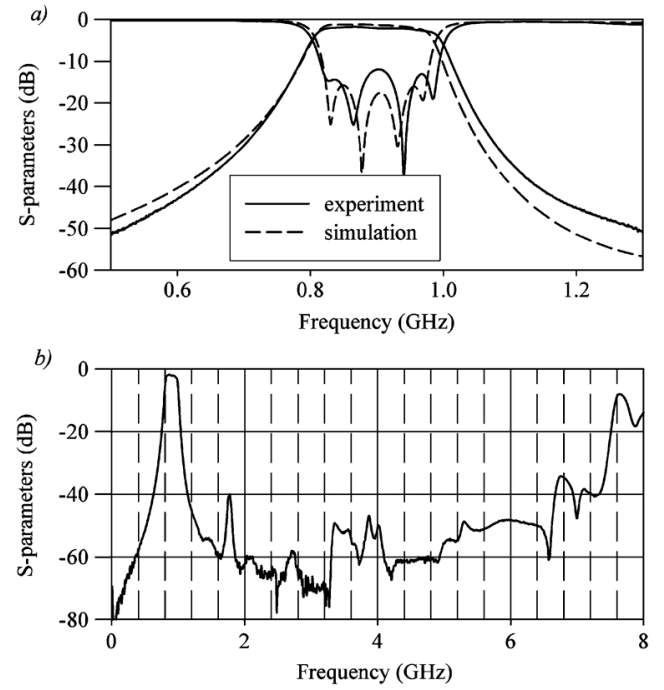

Fig. 5. Frequency responses of the designed (dash line) and fabricated (solid line) filters in the narrow band (a) and the frequency response of the fabricated filter in the wide band (b).

connection of the external resonators (the edge pins) to the feed lines was chosen. In Fig. 4, the fabricated filter is presented.

The comparison of frequency responses obtained for the designed and fabricated filters is represented in the narrow band in Fig. 5(a). The passband is formed by four most low-frequency resonances, one in each interdigital structure (resonator). One can see a good agreement between the simulation and experiment. The only difference between the filters is the wider bandwidth $(21 \%)$ in the fabricated filter instead of $20 \%$ in the designed one. The broadening also brings to the lower return loss $(-13 \mathrm{~dB})$ instead of $-14 \mathrm{~dB}$ in the simulation.

The measured minimum insertion loss in the passband was found to be $1.7 \mathrm{~dB}$, the variation of in-band group delay was measured to be $2 \mathrm{~ns}$. The experimental quality factor of the resonators estimated from frequency response was found to be 80 . It is $30 \%$ lower than simulated value for the single resonator that can be associated with a roughness of resonators boundaries and usage of a solder alloy for the mount whose conductivity is lower than the conductivity of copper.
The fabricated filter was found to have rather wide upper stopband. The frequency response of the fabricated filter is presented in Fig. 5(b) in wider frequency range. At the rejection level higher than $-40 \mathrm{~dB}$, the first spurious passband appears only at $6.6 \mathrm{GHz}$ that corresponds to $7.4 f_{0}$.

\section{CONCLUSION}

A novel microstrip resonator based on interdigital structure is proposed. It consists of two interdigitated microstrip comb structures, and at that the common conductor of each "comb" is connected with the ground along its length. The resonator possesses 2.5 times higher $Q$-factor in comparison with other miniaturized constructions described earlier; this implies from the fact that the high-frequency current at the first resonant frequency flow in the same direction in the pins of interdigital structure. Therefore, the summary inductance of all the pins in the structure has nearly the same magnitude as the inductance of a single pin. Also, the current in the resonator is shared uniformly by all the pins, thus reducing the resistive loss in the resonator.

A 4-pole passband filter was designed and fabricated based on the resonator. It was found that for the central frequency $900 \mathrm{MHz}$ and $20 \%$ fractional bandwidth, the lateral size of the filter is only $10 \mathrm{~mm} \times 4.6 \mathrm{~mm}$. Also, the fabricated filter has upper stopband up to $7.4 f_{0}$ at the level $-40 \mathrm{~dB}$.

\section{REFERENCES}

[1] M. Makimoto and S. Yamashita, "Bandpass filters using parallel coupled stripline stepped impedance resonators," IEEE Trans. Microw. Theory Tech., vol. MTT-28, no. 12, pp. 1413-1417, Dec. 1980.

[2] Y.-T. Lee, J.-S. Lim, C.-S. Kim, D. Ahn, and S. A. Nam, "A compact-size microstrip spiral resonator and its application to microwave oscillator," IEEE Microw. Compon. Lett., vol. 12, no. 10, pp. 375-377, Oct. 2002.

[3] J. Zhu and Z. Feng, "Microstrip interdigital hairpin resonator with an optimal physical length," IEEE Microw. Compon. Lett., vol. 16, no. 12, pp. 672-674, Dec. 2006.

[4] T. Yang, M. Tamura, and T. Itoh, "Compact hybrid resonator with series and shunt resonances used in miniaturized filters and Balun filters," IEEE Trans. Microw. Theory Tech., vol. 58, no. 2, pp. 390-402, Feb. 2010.

[5] C.-H. Liang and C.-Y. Chang, "Novel microstrip stepped-impedance resonator for compact wideband bandpass filters," in Proc. Asia Pacific Microw. Conf. (APMC'09), 2009, pp. 941-944.

[6] Z. Aboush and A. Porch, "Compact narrow bandwidth, lumped element bandstop resonators," IEEE Microw. Compon. Lett., vol. 15, no. 8, pp. 524-526, Aug. 2005.

[7] B. A. Belyaev, S. V. Butakov, N. L. Laletin, A. A. Leksikov, V. V. Tyurnev, and O. N. Chesnokov, "Selective properties of microstrip filters designed on quarter-wavecodirectional hairpin resonators," $J$. Commun. Technol. Electron., vol. 51, no. 1, pp. 20-30, Jan. 2006.

[8] L. Athukorala and D. Budimir, "Compact dual-mode open loop microstrip resonators and filters," IEEE Microw. Compon. Lett., vol. 19, no. 11, pp. 698-700, Nov. 2009.

[9] M. G. Banciu, R. Ramer, and A. Ioachim, "Compact microstrip resonators for $900 \mathrm{MHz}$ frequency band," IEEE Microw. Compon. Lett., vol. 13, no. 5, pp. 175-177, May 2003.

[10] J. S. Hong and M. J. Lancaster, "Capacitively loaded microstrip loop resonator," Electron. Lett., vol. 30, no. 18, pp. 1494-1495, Sep. 1994.

[11] B. S. Virdee and C. Grassopoulosf, "Folded microstrip resonator," in IEEE MTT-S Int. Dig., 2003, pp. 2161-2164.

[12] B. A. Belyaev, A. M. Serzhantov, V. V. Tyurnev, Y. F. Bal'va, and A. A. Leksikov, "Planar bandpass filter with 100-db suppression up to tenfold passband frequency," Progress Electromag. Res. C, vol. 48, pp. 37-44, 2014.

[13] B. A. Belyaev, A. M. Serzhantov, Y. F. Bal'va, A. A. Leksikov, and R. G. Galeev, "A new design of a miniature microstrip resonator with interdigital structure," Tech. Phys. Lett., vol. 40, no. 11, pp. 1010-1013, Nov. 2014. 